\title{
TRATAMENTO DAS NEUROPATIAS DESMIELINIZANTES AQUfLICAS PELO ACTH. RESULTADOS IMEDIATOS E TARDIOS
}

\author{
HORACIO M. CANELAS * \\ JOSÉ ANTONIO LEVY *
}

O tratamento das lesões nervosas dos estados carenciais aquílicos (mieloses funiculares ou degenerações combinadas da medula) ainda é um problema a resolver. $O$ advento da vitamina $B_{12}$ parecia ter solucionado definitivamente a questão, mas prontamente se verificou que essa droga - conquanto superior aos demais medicamentos até então utilizados - representou um progresso relativamente discreto para o esclarecimento do problema. De outro lado, mesmo os resultados hematológicos obtidos nos casos em que as neuropatias aquílicas se acompanham de anemia, nem sempre se mostraram tão brilhantes como se previa ${ }^{4}$. Contudo, os efeitos sôbre as alterações sangüíneas superam sempre, de muito, os resultados neurológicos, o que veio evidenciar mais uma vez a ausência de paralelismo entre os dois setores, fato ainda recentemente salientado por Boudin e col. ${ }^{2}$ e Lafon e col. ${ }^{8}$.

Como já frisamos em trabalhos anteriores ${ }^{3,5}$, é altamente provável que os processos desmielinizantes reconheçam patogenia alérgica, devido aos distúrbios no metabolismo das proteínas alimentares, condicionados primàriamente pela aquilia gástrica. Lembre-se, a propósito, as verificações de Furtado $^{7}$ - confirmadas em parte por Lafon e col. ${ }^{8}$ - de alterações em frações de proteínas plasmáticas em casos de mielose funicular, evidenciadas pelo estudo eletroforético. Tal hipótese patogênica sugeriu-nos o emprêgo do hormônio adrenocorticotrópico nos casos de neuraquilia. Em trabalho anterior ${ }^{5}$, apresentamos os resultados imediatos dêsse tratamento em 4 casos, concluindo que o ACTH revelara uma ação favorável em todos os pacientes.

Todavia, ficamos em dúvida quanto à duração dêsses resultados precoces, visto que, não resolvido o problema fundamental representado pela aquilia, os fatôres patogênicos persistiriam. Tornava-se imprescindível, pois, para a avaliação precisa do método terapêutico, o estudo dos efeitos tardios do hormônio. É o que fazemos agora; neste trabalho - que é, portanto, uma seqüência do anterior ${ }^{5}$ — apresentamos: a) os resultados imediatos dessa terapêutica em 2 novos casos de neuropatias desmielinizantes aquílicas; b) os resultados tardios em 4 casos.

Trabalho da Clínica Neurológica da Fac. Med. da Univ. de São Paulo (Prof. Adherbal Tolosa).

* Assistentes. 


\section{METODO E MATERIAL}

Método — Empregamos o hormônio adrenocorticotrópico (ACTH “Armour") na dose de 12,5 a $15,0 \mathrm{mg}$ diluídos em $500 \mathrm{ml}$ de soluto glicosado a $10 \%$, administrados gôta a gôta na veia, na velocidade média de 20 gôtas/minuto, durante 8 horas. A todos os pacientes foi administrado cloreto de potássio, na dose de 1 a 2 diárias; dieta com restrição de sódio; cuidados habituais no tratamento com êsse hor. mônio. Não foi feita qualquer outra medicação associada. Os pacientes não haviam sido submetidos a qualquer tratamento imediatamente anterior à instituição do ACTH, com exceção dos casos 1 e 2, que receberam anti-histamínicos, sem resultados apreciáveis. No caso 1 instituímos, após 6 meses do término da hormonioterapia, tratamento pela vitamina $B_{1}$ (total de $2,4 \mathrm{~g}$ ); nos casos 2 e 3 empregamos, 6 meses após o ACTH, a vitamina $B_{12}$, na dose de $50 \mathrm{mcg}$ duas vêzes por semana (totais de 1,2 e $2,5 \mathrm{mg}$, respectivamente).

A evolução neurológica foi acompanhada sob ponto de vista semiquantitativo, utilizando-se uma modificação da tabela proposta por Alexander ${ }^{1}$ para o estudo evolutivo de neuropatias de evolução crônica e já referida por nós em trabalho anterior ${ }^{5}$. No estudo clínico demos particular atenção ao exame da sensibilidade vibratória, através da determinação dos niveis raqueanos de apalestesia (pesquisados com diapasão de $256 \mathrm{dv} / \mathrm{s}$ ) e dos limiares de palestesia no hálux, tíbia, dedo anular e olecrânio (pesquisados com o auxílio de um palestesiômetro *).

Material - Os 4 pacientes eram portadores do quadro neurológico característico das neuropatias desmielinizantes aquilicas, avultando, na sintomatologia, as manifestações de lesão dos funículos dorsais e dos nervos periféricos; sinais piramidais discretos foram encontrados em 3 casos. $\mathrm{Em}$ todos foi verificada acloridria gástrica pela prova de Katsch-Kalk. Apenas em um caso havia anemia concomitante, na época da instituiçāo do tratamento pelo ACTH, porém a medula óssea era normoblástica. No caso 2 o mielograma revelou megaloblastose parcial. No caso 3 , o estudo da medula óssea mostrou caracteres superponiveis aos de "carência de fator de maturação eritroblástica" (hiperplasia vermelha com formas intermediárias; presença de células de Tempka e Braun). No caso 4, o mielograma evidenciou anaplasia granulocitica, aumento de eosinófilos; série vermelha com pequena proporção de

\begin{tabular}{|c|c|c|c|c|c|c|c|}
\hline \multirow{2}{*}{$\begin{array}{l}\text { No DO CASO } \\
\text { IDENTIDADB } \\
\text { REG. HC. }\end{array}$} & \multirow{2}{*}{$\begin{array}{c}\text { SEXO. } \\
\text { IDADE } \\
\text { CÔR }\end{array}$} & \multirow{2}{*}{$\begin{array}{l}\text { INICIO DOS } \\
\text { SINTOMAS } \\
\text { (mค8в8) }\end{array}$} & \multirow{2}{*}{$\begin{array}{c}\text { TOTAL DE } \\
\text { ACTH } \\
\text { (m\&) }\end{array}$} & \multicolumn{2}{|c|}{ HEMOGRAMA } & \multicolumn{2}{|c|}{ EATSCE } \\
\hline & & & & Brit. & $\mathrm{Hb}$. & Al & $\mathbf{A T}$ \\
\hline $\begin{array}{c}1 \\
\text { T.L. } \\
287.204\end{array}$ & $\begin{array}{c}F \\
49 \\
B\end{array}$ & 24 & 225,0 & 2,4 & 7,8 & 0 & 10 \\
\hline $\begin{array}{c}2 \\
\text { J.P.M. } \\
276.884\end{array}$ & $\begin{array}{c}11 \\
46 \\
B\end{array}$ & 14 & 252,5 & 4,2 & 11,0 & 10 & 16 \\
\hline $\begin{array}{c}3 \\
\text { G.C. } \\
030.982\end{array}$ & $\begin{array}{c}M \\
43 \\
B\end{array}$ & 84 & 390,0 & 4,2 & 13,3 & 0 & 8 \\
\hline $\begin{array}{c}\text { A. P. } \\
331.359\end{array}$ & $\begin{array}{c}F \\
55 \\
B\end{array}$ & 36 & 350,0 & 3,7 & 12,0 & 0 & .8 \\
\hline
\end{tabular}

Quadro 1 - Material clínico (Erit., eritrócitos em milhões por $\mathrm{mm}^{3}$; $\mathrm{Hb}$., hemoglobina em $\mathrm{g} / 100 \mathrm{ml} ; A L$, acidez livre; $A T$, acidez total). 


\begin{tabular}{|c|c|c|c|c|c|c|c|c|c|c|c|c|c|}
\hline \multirow{2}{*}{\multicolumn{2}{|c|}{$\begin{array}{l}\text { SINTOMATOLOGIA } \\
\text { NEUROLOGICA }\end{array}$}} & \multicolumn{3}{|c|}{ CASO 1} & \multicolumn{3}{|c|}{ CASO 2} & \multicolumn{3}{|c|}{ CASO 3} & \multicolumn{3}{|c|}{ CASO 4} \\
\hline & & $\begin{array}{l}\text { Antes } \\
\text { do ACTH }\end{array}$ & $\begin{array}{l}\text { Final } \\
\text { do ACTH }\end{array}$ & $\begin{array}{l}\text { Result. } \\
\text { tardio* }\end{array}$ & $\begin{array}{l}\text { Antes } \\
\text { do ACTH }\end{array}$ & $\begin{array}{l}\text { Final } \\
\text { do ACTH }\end{array}$ & $\begin{array}{l}\text { Result. } \\
\text { tardio }\end{array}$ & $\begin{array}{c}\text { Antes } \\
\text { do ACTH }\end{array}$ & $\begin{array}{l}\text { Final } \\
\text { do ACTH }\end{array}$ & $\begin{array}{l}\text { Result. } \\
\text { tardio*** }\end{array}$ & $\begin{array}{l}\text { Antes } \\
\text { do ACTH }\end{array}$ & $\begin{array}{c}\text { Final } \\
\text { do ACTH }\end{array}$ & $\begin{array}{l}\text { Result. } \\
\text { tardio*t }\end{array}$ \\
\hline \multirow{2}{*}{ 品 } & Deficit. & 4 & 0 & 0 & 8 & 4 & 4 & 8 & 8 & 0 & 16 & 0 & 0 \\
\hline & Libert. & 0 & 0 & 0 & 10 & 30 & 10 & 0 & 0 & 0 & 10 & 10 & 20 \\
\hline \multirow{4}{*}{ 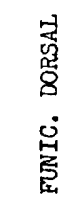 } & Eq.estát. & 15 & 5 & 15 & 15 & 15 & 15 & 15 & 5 & 5 & 5 & 5 & 5 \\
\hline & Eq.dinām. & 15 & 5 & 0 & 15 & 10 & 15 & 5 & 5 & 5 & 0 & 0 & 0 \\
\hline & Coord.ap. & 10 & $l_{+}$ & 0 & 14 & 10 & 10 & 0 & 0 & 0 & 0 & 0 & 0 \\
\hline & Artrest. & 0 & 0 & 0 & 15 & 10 & 10 & 10 & 0 & 0 & 10 & 0 & 10 \\
\hline \multirow{2}{*}{ 逭 } & Palest.R. & 18 & 18 & 7 & 5 & 4 & 4 & 13 & 12 & 7 & 19 & 11 & 9 \\
\hline & Palest.I. & 11,5 & 9,5 & 12,5 & 6,5 & 6,0 & 11,5 & 8,5 & 7,5 & 9,5 & 8,0 & 7,0 & 8,0 \\
\hline \multirow{5}{*}{ 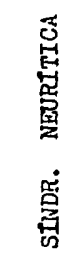 } & Hipotonia & 4 & 4 & 0 & 14 & 4 & 4 & 0 & 0 & 0 & 4 & 4 & 4 \\
\hline & Hipo-Arrefl. & 20 & 8 & 4 & 2 & 2 & 2 & 29 & 29 & 29 & 0 & 0 & 0 \\
\hline & Hiperalg. & 0 & 0 & 0 & 10 & 4 & 4 & 2 & 2 & 0 & 10 & 0 & 4 \\
\hline & Parestesias & 20 & 20 & 20 & 10 & 4 & 10 & 4 & 4 & 4 & 20 & 6 & 14 \\
\hline & Hipoest.sup. & 0 & 0 & 10 & 0 & 0 & 0 & 10 & 10 & 10 & 50 & 50 & 30 \\
\hline \multicolumn{2}{|c|}{ COMPUTO TOTAL } & 117,5 & 73,5 & 68,5 & 114,5 & 83,0 & 99,5 & 104,5 & 82,5 & 69,5 & 152,0 & 33,0 & 94,0 \\
\hline \multirow{2}{*}{\multicolumn{3}{|c|}{$\begin{array}{l}\text { CÔMPUTO MẼIO } \\
\text { MBLHORA PERCENTUAL NEDIA }\end{array}$}} & \multicolumn{4}{|c|}{ ANTES DO ACTH } & \multicolumn{3}{|c|}{ FINAL DO ACTH } & \multicolumn{4}{|c|}{ RESULT.TARDIO } \\
\hline & & & \multicolumn{3}{|c|}{112,12} & & & \multicolumn{2}{|l|}{$\begin{array}{c}83,00 \\
26 \%\end{array}$} & \multicolumn{4}{|c|}{82,88} \\
\hline
\end{tabular}

Quadro 2 - Avaliação semiquantitativa dos resultados imediatos e tardios (*, 6 meses; **, 3 meses) do emprêgo do ACTH em nossos casos. Eq., equilibrio; coord.ap., coordenação apendicular ; palest.R., palestesia na raque; palest.L., limiar de palestesia. 
macroeritroblastos; maturação conservada; megacariócitos normais. Verificou-se, em 3 casos, a presença de eosinofilia, apesar da negatividade de cuidadosos exames de fezes, no sentido de comprovar uma verminose; talvez êste fato reforce a hipótese da patogenia alérgica do processo neuropatológico, que justificou a terapéutica por nós adotada. As características fundamentais do material clínico estão representadas no quadro 1; verifica-se que todos os pacientes apresentavam sintomas mais de 12 meses antes do inicio da hormonioterapia.

\section{RESULTADOS}

A avaliação semiquantitativa das manifestaçōes neurológicas, feita por meio da tabela já referida, está representada no quadro 2, onde referimos os resultados logo após o tratamento pelo ACTH e os comprovados tardiamente (de 3 a 6 meses após o término dessa terapêutica). Verifica-se o vulto que assumiram os distúrbios devidos à lesão funicular dorsal, fato, aliás, já bem conhecido. Como se pode deduzir do quadro 3 , na sintomatologia prévia ao tratamento, a sindrome funicular dorsal contribuiu com $49,8 \%$ dos sinais, ao passo que a síndrome neuritica representou $39,9 \%$ e a síndrome piramidal, $11,2 \%$.

Como se observa pelo exame do quadro 2, os resultados imediatos do tratamento pelo hormônio adrenocorticotrópico foram satisfatórios em todos os casos. Devemos salientar particularmente os resultados obtidos nos casos 3 e 4, porquanto os casos 1 e 2 já constaram de trabalho anterior ${ }^{5}$. Dentre as três sindromes neurológicas, a mais beneficiada foi a piramidal, sendo mais resistente a síndrome neuritica (quadro 3 ).

\begin{tabular}{|c|c|c|c|c|c|c|c|c|c|}
\hline \multirow[b]{2}{*}{ CASO } & \multicolumn{3}{|c|}{ SIMDROME FUNICULAR DORSAL } & \multicolumn{3}{|c|}{ SINDROME PIRAMIDAL } & \multicolumn{3}{|c|}{ SINDROME NEURITICA } \\
\hline & $\begin{array}{c}\text { Antes } \\
\text { do ACTh }\end{array}$ & $\begin{array}{c}\text { Final } \\
\text { do ACTH }\end{array}$ & $\mid \begin{array}{l}\text { Result. } \\
\text { tardio }\end{array}$ & $\begin{array}{l}\text { Antes } \\
\text { do ACTII }\end{array}$ & $\begin{array}{c}\text { Final } \\
\text { do ACTH }\end{array}$ & $\begin{array}{l}\text { Result. } \\
\text { tardio }\end{array}$ & $\begin{array}{l}\text { Antes } \\
\text { do ACTh }\end{array}$ & $\begin{array}{c}\text { Final } \\
\text { do AC'II }\end{array}$ & $\begin{array}{l}\text { Result. } \\
\text { trrdio }\end{array}$ \\
\hline 1 & 69,5 & 41,5 & 34,5 & 4,0 & 0 & 0 & 44,0 & 32,0 & 34,0 \\
\hline 2 & 70,5 & 55,0 & 65,5 & 18,0 & 14,0 & 14,0 & 26,0 & 14,0 & 20,0 \\
\hline 3 & 61,5 & 29,5 & 26,5 & 8,0 & 8,0 & 0 & 45,0 & 45,0 & 43,0 \\
\hline 4 & 42,0 & 23,0 & 32,0 & 26,0 & 10,0 & 10,0 & 84,0 & 60,0 & 52.0 \\
\hline TOTAL & 243,5 & 149,0 & 158,5 & 56,0 & 32,0 & 24,0 & 199,0 & 151,0 & 149,0 \\
\hline $\begin{array}{l}\text { MSLHORA } \\
\text { MEDIA }\end{array}$ & - & $38,9 \%$ & $33,3 \%$ & - & $41,4 \%$ & $67,1 \%$ & - & $24,1 \%$ & $24,5 \%$ \\
\hline
\end{tabular}

Quadro 3 - Resultados imediatos e tardios em nossos casos, relacionados às três sindromes neurológicas.

O estudo dos resultados tardios evidencia marcante uniformidade nos 4 casos, revelando que os efeitos obtidos logo após o término do tratamento se mantêm por período relativamente longo. É deveras notável a identidade das cifras de melhora percentual média, nos cômputos imediato e tardio $(26 \%)$. Analisando separadamente as três sindromes neurológicas verificamos, contudo, que houve: a) igualdade entre os resultados imediatos e tar- 
dios no tocante à sindrome neuritica; b) melhora da síndrome piramidal após o período de seguimento; c) discreta piora dos resultados tardios em relação aos imediatos, no tocante à sindrome funicular dorsal.

A instituição de tratamento pela vitamina $B_{1}$ no caso 1 e pela vitamina $B_{12}$ (em alta dose total) nos casos 2 e 3 , após um período de observação pós-ACTH de 6 meses, não modificou em nada o quadro clínico, como se comprova pelo exame do gráfico 1 .

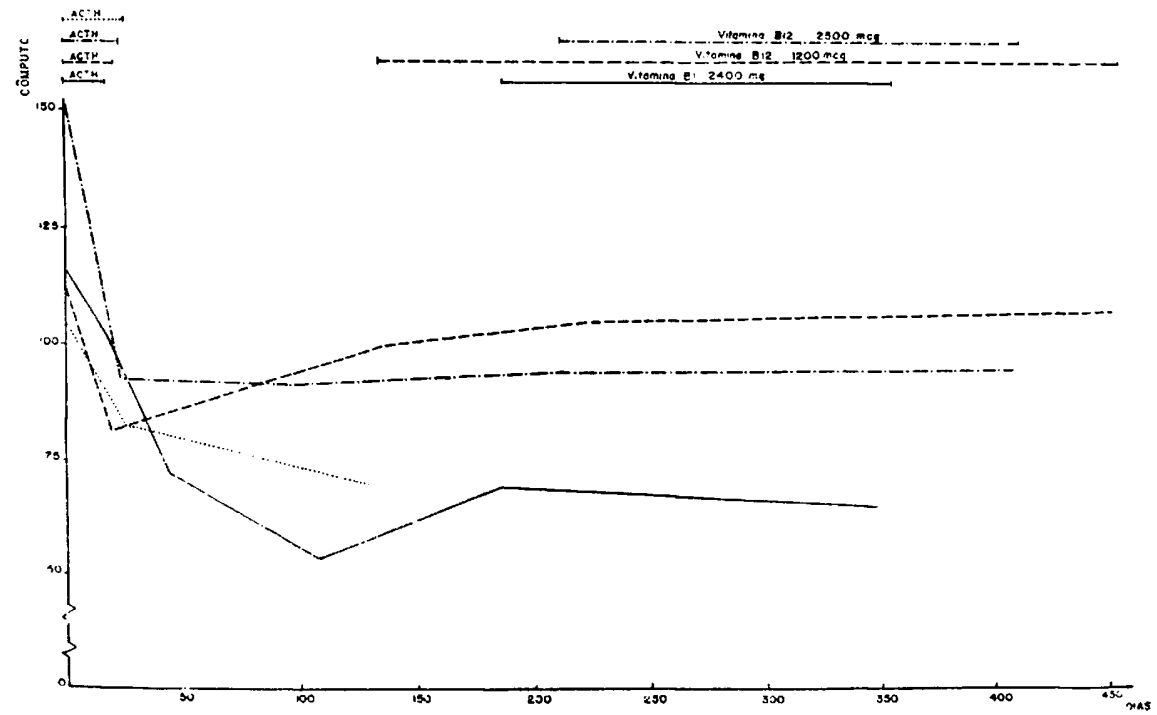

Gráfico 1 - Representação da evolução neurológica, de acôrdo com os dados do quadro 2, e após o emprêgo de vitaminas $B_{1}$ e $B_{12}$ (em linha contínua, caso 1; em linha interrompida, caso 2; em pontilhado, caso 3; em traço e ponto, caso 4).

Apesar de ser pequeno nosso material clínico, comprova-se que o emprêgo do hormônio adrenocorticotrópico nas neuropatias desmielinizantes aquílicas resultou em apreciável melhora do quadro clínico, a qual se manteve durante um periodo variável entre 3 e 6 meses, não tendo sido modificada pelo emprêgo ulterior de outras medicações (vitaminas $B_{1}$ e $B_{12}$ ).

Acreditamos que esta nossa contribuição para a terapêutica das assim chamadas degenerações combinadas da medula tem cunho de originalidade, pois não encontramos, em cuidadosa revisão da literatura, qualquer referência ao emprêgo do ACTH nesses casos. Mesmo com relação à anemia perniciosa, as referências à utilização dêsse hormônio ou da cortisona são muito escassas ${ }^{6}$, merecendo citação apenas Wintrobe e col. ${ }^{9}$ que, em 2 casos de moléstia de Addison-Biermer, verificaram que a administração de ACTH provocou reticulocitose irregular em platô, mas não se acompanhou de redução da anemia ou melhora clínica. 


\section{RESUMO}

Os autores apresentam os resultados imediatos e tardios do emprêgo do ACTH em 4 casos de neuropatias desmielinizantes aquílicas (assim chamadas degeneraçōes combinadas da medula). Os efeitos imediatos foram satisfatórios em todos os casos, particularmente em relação à síndrome piramidal, mostrando-se mais resistente a síndrome neuritica. Os efeitos tardios foram, no conjunto, absolutamente idênticos aos imediatos, demonstrando que os benefícios se mantiveram por períodos de 3 a 6 meses; a análise separada das sindromes neurológicas evidenciou melhora ulterior dos sinais piramidais, estabilidade da sintomatologia neurítica e discreta recidiva da sindrome funicular dorsal. A instituição de tratamentos pelas vitaminas $B_{1}$ e $B_{12}$, em altas doses, após um período pós-ACTH de 6 meses (em 3 casos) não modificou o quadro clínico.

\section{SUMMARY \\ Treatment of achylic demyelinating neuropathy with ACTH; early and late results.}

The authors report the early and late results of the use of ACTH in 4 cases of achylic demyelinating neuropathy (so-called subacute combined degeneration of the spinal cord). Daily doses of 12.5 or $15.0 \mathrm{mgm}$. of corticotropin were given by continuous drip method, without any other associated drug which could interfere in the results. By employing a semi-quantitative method for evaluation of the clinical effects, an average improvement of 26 per cent was found, the same results being observed soon after the treatment and by a follow-up from 3 to 6 months. The effects on the pyramidal syndrome were particularly satisfactory both early and lately; the improvement of the dorsal funicular syndrome was not so long lasting. Treatment with vitamins $B_{1}$ and $B_{12}$ six months after the use of $A C T H$, in 3 cases, caused no further change in the clinical picture.

\section{BIBLIOGRAFIA}

1. ALEXANDER, L. - New conceppt of critical steps in course of chronic debilitating neurologic disease in evaluation of therapeutic response. Arch. Neurol. a. Psychiat., 66:253-271 (setembro) 1951. 2. BOUDIN, G.; BARBIZET, J.; LABET, R. Les neuropathies achyliques. Rev. Neurol., 91:361-366, 1954. 3. CANELAS, H. M. Distúrbios neurológicos nos estados carenciais aquilicos. Arq. Neuro-Psiquiat., 10:140 (março) 1952. 4. CANELAS, H. M.; JAMRA, M. A. - Estudo comparativo dos efeitos hematológicos e neurológicos do extrato hepático, ácido fólico e vitamina $\mathrm{B}_{12}$ no tratamento da anemia perniciosa de Addison-Biermer. Arq. Neuro-Psiquiat., 11: 229-246 (setembro) 1953. 5. CANELAS, H. M.; LEVY, J. A. - Resultados preliminares do emprêgo do ACTH nas degenerações combinadas da medula. Arq. Neuro-Psiquiat., 11:253-258 (setembro) 1953. 6. a) COSTE, F.; MALLARMÉ, J.; BOUREL, M. - La place de la corticothérapie (ACTH et cortisone) dans le traitement des hémopathies. Ann. de Méd., 54:377-430, 1953; b) GARDNER, F. - Discussão do trabalho: ACTH in leukemia. Blood, 5:791 (agôsto) 1950; c) HEILMEYER, L. - ACTH 
und Cortisontherapie bei nicht leukhämischen Bluterkrankungen. Acta Haematol., 7: 206-216 (abril) 1952; d) MERRITT, H. H. - Corticotropin and cortisone in diseases of the nervous system. Yale J. Biol, a. Med., 24:466-473 (junho) 1952. 7. FURTADO, D. - Etudes sur la myélose funiculaire. I: Tableau clinique, d'après une centaine d'observations. II: Rôle d'une protéine anormale dans la pathogénie. Rev. Neurol., 90:82-94, 1954. 8. LAFON, R.; PAGES, P.; LABAUGE, R.; TEMPLE, J.-P. - Le syndrome neuro-anachlorhydrique. A propos de 31 observations. Rev. Neurol., 91:321329, 1954. 9. WINTROBE, M. M.; CARTWRIGHT, G. E.; KUHNS, W. J.; PALMER, J. G.; LAHEY, M. E. - The effects of ACTH on the hematopoietic system. Blood, 5:789-790 (agôsto) 1950.

Clinica Neurológica. Hospital das Clinicas da Fac. Med. da Univ. de São PauloCaixa Postal 3461 - São Paulo, Brasil. 